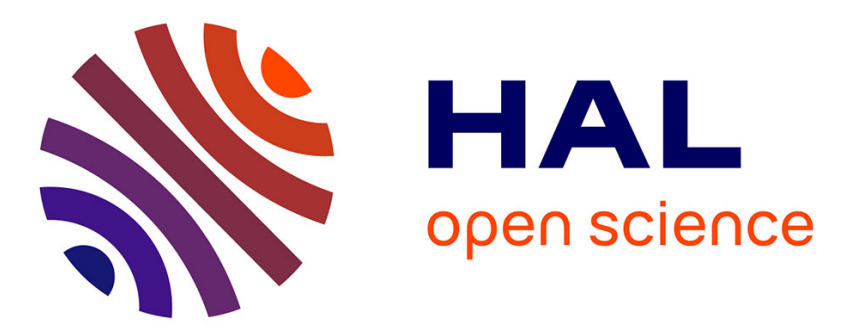

\title{
GALEN: A Geometric Framework for Global Optimal Power Allocation in a Full Duplex D2D Network
}

\author{
Hussein Chour, Faouzi Bader, Youssef Nasser, Oussama Bazzi
}

\section{To cite this version:}

Hussein Chour, Faouzi Bader, Youssef Nasser, Oussama Bazzi. GALEN: A Geometric Framework for Global Optimal Power Allocation in a Full Duplex D2D Network. IEEE Wireless Communications and Networking Conference (WCNC'2019), Apr 2019, Marrakech, Morocco. 10.1109/WCNC.2019.8885846 . hal-02002848

\section{HAL Id: hal-02002848 \\ https://hal.science/hal-02002848}

Submitted on 22 Apr 2019

HAL is a multi-disciplinary open access archive for the deposit and dissemination of scientific research documents, whether they are published or not. The documents may come from teaching and research institutions in France or abroad, or from public or private research centers.
L'archive ouverte pluridisciplinaire HAL, est destinée au dépôt et à la diffusion de documents scientifiques de niveau recherche, publiés ou non, émanant des établissements d'enseignement et de recherche français ou étrangers, des laboratoires publics ou privés. 


\title{
GALEN: A Geometric Framework for Global Optimal Power Allocation in a Full Duplex D2D Network
}

\author{
Hussein CHOUR ${ }^{1,2}$, Faouzi BADER ${ }^{1}$, Youssef NASSER ${ }^{3}$, and Oussama BAZZI ${ }^{2}$ \\ ${ }^{1}$ CentraleSupélec/IETR, CentraleSupélec Campus de Rennes, 35510 Cesson-Sévigné, France \\ ${ }^{2}$ Physics and Electronics Department, Faculty of Science 1, Lebanese University, Beirut, Lebanon \\ ${ }^{3}$ ECE Department, American University of Beirut, Bliss Street, Beirut, Lebanon, \\ Email:\{hussein.chour@supelec.fr, faouzi.bader@supelec.fr, yn10@aub.edu.lb, obazzi@ul.edu.lb, \}
}

\begin{abstract}
This paper studies the rate maximization problem of a Full duplex(FD) D2D underlaying cellular network. In the considered scenario, multiple FD-D2D pairs coexist with multiple cellular users which generate mutual interference between the two communication types. The complicated interference environment makes the optimization problem a non-concave problem. To solve this problem, we discuss how to leverage the monotonic optimization theory to obtain the global optimal solution at the cost of high complexity. We also derive a geometric based optimization framework, denoted as GALEN, that achieves the global optimality with much lower complexity, and also it provides a closed form expression for the solution. The simulation results show the importance of the proposed solution. Index Termsfull-duplex transmission, device-to-device (D2D) communication, optimal power allocation, duplex mode selection
\end{abstract}

\section{INTRODUCTION}

Given the scarcity property of the cellular radio spectrum, coupled with the rapidly growing of the users' data traffic demands [1], the need of new technologies which efficiently use the radio resources and fulfill the customers' demands has become an essential part in the upcoming cellular network (5G). In this context, Full Duplex (FD) and device-to-device (D2D) communication are proposed to enhance the radio spectrum efficiency and the users' experience in a cellular network. D2D allows two nearby devices to bypass the base station (BS) and communicate directly peer-to-peer as a D2D communication pair [2]. On the other hand, FD allows a device to simultaneously transmit and receive in the same frequency band at the same time slot [3].

Due to the short distance feature of the D2D communication, integrating the FD technology with D2D communication can further enhance the radio spectrum efficiency and the users' throughput [4]. However, the practical FD devices add new challenges on both the D2D communication and the traditional cellular communication. For instance, the existing FD transceivers can not perfectly remove the self interference (SI) imposed on the receiver by the node's own transmitter [3]. Thus, the residual self interference (RSI) which is tightly related to the transmitter power value highly affects the performance of FD transmission. In addition, using the FD technique creates additional interference in the network which may overwhelm the conventional cellular link. Thus, a proper power allocation is needed to exploit the benefits of FD and guarantee the quality of service (QoS) of the users.

The authors of [5] tackled the power allocation problem of an isolated full duplex D2D pair operating on the cellular resources. Motivated by the work of [5], the authors of [6] aimed to maximize the throughput of a FD-D2D link by properly allocating the power of the D2D pair and the CU while satisfying the minimum rate requirement of the CU. The ergodic rate of a FD-D2D pair coexisting with a CU was derived and analysed in [7] considering a fixed transmission power strategy. The authors of [8] proposed to maximize the ergodic sum-rate of a FD-D2D pair underlaying cellular network while satisfying the data-rate requirement of the CUs. In [9], a power allocation solution was proposed by using the DC programming theory [10]. However, none of the above works provided a global optimal power allocation scheme. Indeed, due to the complicated interference environment generated by the FD transmission, the power allocation is a nonconcave problem. Thus finding the global optimal solution is a challenging task. Instead, a first-order optimal solution was given in literature (e.g. [9]), or a sub-optimal solution was provided as in [5], [7], [8].

In this paper, we aim at providing the global optimal solution of the rate maximization problem of a FD-D2D network. To that end, we develop two novel optimization frameworks for a FD-D2D underlaying cellular network. The former provides the global optimal solution by means of monotonic optimization theory (MO) [11], [12] at the expense of high complexity, whereas the latter achieved the global optimality by exploiting the geometric structure of the feasible set with much reduced complexity. For the sake of readability, we named the second approach GALEN (Geometrical framework Approach for gLobal optimal powEr allocatioN). The major contribution of this work are as follows:

- Shedding the light on the optimal rate gain of a FD-D2D network

- Providing two approaches that lead to the global optimal 
solution. To the best of the authors knowledge, none of the existing works provides the global optimal solution of a FD-D2D network. Global optimality is achieved by exploiting the MO theory and developing a new geometric based optimization framework.

- Providing a closed form expression for the optimal power allocation

- Highlighting the impact of the SI cancellation factor and the D2D proximity distance on the throughput gain.

The rest of this paper is organized as follows. Section II describes the system model and formulates the optimization problem. Section III derives the optimal solution by using the MO theory while section IV derives the optimal solution by using the geometric structure of the feasible set. The simulation results are given in section $\mathrm{V}$, and finally, the conclusion is drawn in section VI.

\section{SYSTEM MODEL AND PROBLEM FORMULATION}

We consider a D2D communication underlaying cellular network where $M$ FD-D2D pairs coexist with $M$ half duplex cellular users. Without loss of generality, we will consider in this work that each D2D pair will share the same resources as an uplink $\mathrm{CU}$ resource. We will denote that resource as $j$. In other words, Each D2D pair $D 2 D_{j}$ consists of two nearby devices, denoted as $D_{1}^{j}$ and $D_{2}^{j}$, that have been assigned the uplink (UL) resources of the $j$ th cellular users $\left(C U_{j}\right)$. UL sharing is particularly considered since the UL resources are underutilized compared to that of downlink. Furthermore, sharing the UL spectrum only affects the BS and incurred interference can be handled by BS coordination.

An instance of this network is drawn in Fig.1 in which two D2D pairs coexist with two cellular users $(M=2)$. The channel gain between the $j$ th cellular user and the BS is denoted as $g_{c, b s}^{j}$ while the channel gain between $D_{1}^{j}$ and $D_{2}^{j}$ and between $D_{2}^{j}$ and $D_{1}^{j}$ are denoted as $g_{d}$. $h_{d 1, b s}^{j}$ and $h_{d 2, b s}^{j}$ respectively denotes the interference channel gains from $D_{1}^{j}$ and $D_{2}^{j}$ to BS. $h_{c, d 1}^{j}$ and $h_{c, d 2}^{j}$ denotes the interference channel gains from the $j$ th cellular user to $D_{1}^{j}$ and $D_{2}^{j}$ respectively. All direct/interference channels are assumed to be zero-mean complex Gaussian random variables (i.e., channels are expressing Rayleigh fading) with variance $l_{i j}$, where $i \in\{c ; d 1 ; d 2\}$, $j \in\{b s ; d 1 ; d 2\}, i \neq j, l_{i j}$ denotes the distance between the nodes $i$ and $j$, and $\alpha$ denotes the path loss exponent. $h_{1,1}^{j}$ and $h_{2,2}^{j}$ stands for the residual self interference at $D_{1}^{j}$ and $D_{2}^{j}$. They can be modeled as complex Gaussian random variables with zero-mean and variance $\eta P_{d 1}^{j}$ and $\eta P_{d 2}^{j}$ respectively [5], [6], [13], where $P_{d 1}^{j}$ and $P_{d 2}^{j}$ are respectively the transmission power of $D_{1}^{j}$ and $D_{2}^{j}, \eta$ denotes the SI cancellation capability of each D2D device.

\section{A. Full duplex D2D link's rate}

In the FD D2D communication mode, both $D_{1}^{j}$ and $D_{2}^{j}$ reuse the spectrum of $C U_{j}$ to communicate with each other. By denoting $N_{0}$ the power of the additive white Gaussian noise, and $P_{c}^{j}$ the transmission power of the $j$ th cellular user

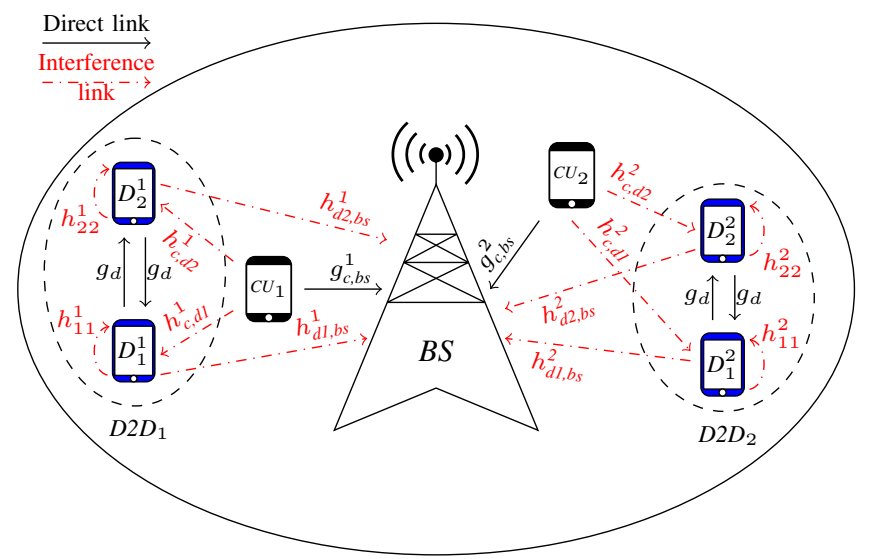

Fig. 1. Full duplex D2D pairs sharing the UL resources of the cellular users. then the received signal to interference plus noise ratio (SINR) at the BS, at $D_{1}^{j}$ and at $D_{2}^{j}$ when $D 2 D_{j}$ shares the spectrum of $C U_{j}$ can be respectively expressed as

$$
\begin{aligned}
\Gamma_{b s}^{j} & =\frac{P_{c}^{j} g_{c, b s}^{j}}{P_{d 1}^{j} h_{d 1, b s}^{j}+P_{d 2}^{j} h_{d 2, b s}^{j}+N_{0}} \\
\Gamma_{d 1}^{j} & =\frac{P_{d 2}^{j} g_{d}^{j}}{P_{c}^{j} h_{c, d 1}^{j}+\eta P_{d 1}^{j}+N_{0}} \\
\Gamma_{d 2}^{j} & =\frac{P_{d 1}^{j} g_{d}^{j}}{P_{c}^{j} h_{c, d 2}^{j}+\eta P_{d 2}^{j}+N_{0}}
\end{aligned}
$$

Applying Shannon theorem, the rate of $D 2 D_{j}\left(R_{f d}^{j}\right)$ can be expressed as the summation of $D_{1}^{j}$,s rate $\left(R_{d 1}^{j}\right)$ and $D_{2}^{j}$,s rate $\left(R_{d 2}^{j}\right)$.

$$
R_{f d}^{j}\left(\mathbf{p}_{j}\right)=\underbrace{B_{j} \log _{2}\left(1+\Gamma_{d 1}^{j}\right)}_{R_{d 1}^{j}}+\underbrace{B_{j} \log _{2}\left(1+\Gamma_{d 2}^{j}\right.}_{R_{d 2}^{j}})
$$

with $\mathbf{p}_{j}=\left(P_{d 1}^{j}, P_{d 2}^{j}, P_{c}^{j}\right)$ being the transmission power of the couple $\left(D 2 D_{j}, C U_{j}\right) . B_{j}$ denotes the bandwidth of $C U_{j}$ which is reused by $D 2 D_{j}$.

\section{B. Problem formulation}

The main rationale behind introducing D2D communications is to mitigate the heavy data traffic in the cellular network. In this paper, we aim at maximizing the total D2D capacity while satisfying the QoS of the cellular users ${ }^{1}$. Let $r_{\text {min }}^{j}$ be the minimum data rate of $C U_{j}$, and denote by $\mathbf{P}=\left[\mathbf{p}_{j}\right]_{M \times 3}$ the matrix of the users' transmission power, the D2D sum-rate maximization problem can be formulated as

$$
\begin{aligned}
& \boldsymbol{P 1}: \max _{\mathbf{P}} \sum_{j=1}^{M} R_{f d}^{j} \text { s.t. } \mathbf{P} \in \Phi \\
& \Phi=\left\{\Gamma_{b s}^{j} \geq \gamma_{\min }^{j}=2^{r_{\text {min }}^{j}}-1, j \in\{1, \ldots, M\}\right. \\
& \left.0 \leq P_{i}^{j} \leq P_{\max }^{i}, i \in\{d 1, d 2, c\}, j \in\{1, \ldots, M\}\right\},
\end{aligned}
$$

where $P_{\max }^{i}$ is the maximum transmission power of a transmitter $i$, and $\gamma_{\min }^{j}$ is the minimum required SINR level to achieve $r_{\text {min }}^{j}$. Solving $\boldsymbol{P 1}$ is the target of the next section.

\footnotetext{
${ }^{1}$ The QoS of the CU sharing the resource might be highly constrained
} 


\section{Reformulation of problem $\boldsymbol{P 1}$}

First we observe that the couples $\left(D 2 D_{j}, C U_{j}\right), j \in$ $\{1, \ldots, M\}$, are independent from each others, and thus $\boldsymbol{P I}$ can be rewritten as:

$$
\boldsymbol{P 2}: \sum_{j=1}^{M} \max _{\mathbf{p}_{j}} R_{f d}^{j} \text { s.t. } \mathbf{p}_{j} \in \Phi .
$$

Next, by using the Logarithm's properties, we rewrite $R_{f d}^{j}$ as:

$$
R_{f d}^{j}\left(\mathbf{p}_{j}\right)=B_{j} \log _{2}\left(Q_{j}\left(\mathbf{p}_{j}\right)\right)=B_{j} \log _{2}\left(\left(1+\Gamma_{d 1}^{j}\right)\left(1+\Gamma_{d 2}^{j}\right)\right) .
$$

The Logarithm is a monotonic increasing function, and thus maximizing $R_{f d}^{j}\left(\mathbf{p}_{j}\right)$ is equivalent to maximize $Q_{j}\left(\mathbf{p}_{j}\right)$. Therefore, $\boldsymbol{P} \mathbf{2}$ can be equivalently written as:

$$
\begin{aligned}
& \boldsymbol{P 3}: \sum_{j=1}^{M} R_{f d}^{j}\left(\mathbf{p}_{j}^{\star}\right) \\
& \text { such that } \mathbf{p}_{j}^{\star}=\underset{\mathbf{p}_{j} \in \Phi}{\operatorname{argmax}} Q_{j}\left(\mathbf{p}_{j}\right)
\end{aligned}
$$

with $\mathbf{p}_{j}^{\star}=\left(P_{d 1}^{j, \star}, P_{d 1}^{j, \star}, P_{c}^{j, \star}\right)$ being the optimal power allocation vector for the couple $\left(D 2 D_{j}, C U_{j}\right)$.

Now, observe that $Q_{j}\left(\mathbf{p}_{j}\right)$ is a non-concave function (since it is the multiplication of two linear fractional functions). Hence, $\boldsymbol{P 3}$ is a non-concave optimization problem. Thus, solving $\boldsymbol{P 3}$ is a challenging task. In the following we provide two approaches to solve $\boldsymbol{P 3}$. The first approach finds the global optimal solution by using the monotonic optimization theory at the expense of high complexity. The second approach utilizes the geometrical structure of the feasible set to find the global optimal solution with much lower complexity, and it is denoted as GALEN. Moreover, GALEN provides a closed form expression for the optimal solution which make it very useful for resource allocation.

\section{OPTIMAL POWER ALLOCATION BY MONOTONIC OPTIMIZATION}

In this section, we focus on finding the optimal solution of $\boldsymbol{P 3}$, the equivalent problem of $\boldsymbol{P 1}$, with the assumption that the BS has perfect knowledge about the channel state information for both the cellular and the D2D users.

In general, globally solving a non-convex optimization problem can involve examining every point in the feasible set. Contrarily, monotonic optimization theory exploits the monotonicity property of the utility function and the constraints in the optimization problem in a much more efficient way but still with exponential complexity in the number of optimization variables. The key idea behind monotonic optimization theory is to search for the global solution on the outer boundary of the feasible set instead of exploring the complete feasible set of the problem. Thus, the usage of monotonic optimization theory is limited to the problems which have certain properties. In particular, the monotonic optimization tool can solve the general problem $\max _{x \in \mathcal{G} \cap \mathcal{H}} f(x)$ providing that the following three properties hold true [14, Chapter I].
- $f(x)$ is an increasing function;

- $\mathcal{G}$ is a normal set;

- $\mathcal{H}$ is a co-normal set.

A function $f: \mathbb{R}^{n} \mapsto \mathbb{R}$ is called an increasing function if for any two vectors $\mathbf{x} \in \mathbb{R}^{N}$ and $\mathbf{y} \in \mathbb{R}^{N}$ we have $f(x) \preceq$ $f(y)$ when $0 \preceq x \preceq y^{2}$. A set $\mathcal{G} \subset \mathbb{R}^{n}$ is normal (conormal) set, if for any point $x \in \mathcal{G}$, all other points $x^{\prime}$ such that $x^{\prime} \leq x\left(x^{\prime} \geq x\right)$ are also in the set $\mathcal{G}$. Based on the above requirements $\mathrm{MO}$ appears to be non-applicable to $P 3$ (Since $Q_{j}$ is non-increasing with $\mathbf{p}_{j}$ ). In the following we show how to transform $P 3$ into an MO problem.

It is first straightforward to claim that $\Gamma_{d 1}^{j}\left(\mathbf{p}_{j}\right), \Gamma_{d 2}^{j}\left(\mathbf{p}_{j}\right)$, and $\Gamma_{b s}^{j}\left(\mathbf{p}_{j}\right)$ are always strictly positive. Now let $\Gamma_{j}=$ $\left(\Gamma_{d 1}^{j}, \Gamma_{d 2}^{j}, \Gamma_{b s}^{j}\right)$ be the achieved SINR vector for all links, it is clear that the function $Q\left(\Gamma_{j}\right)$ is an increasing function with $\Gamma_{j}$. Thus, the optimization problem defined in (10) has a hidden monotonocity structure which can be extracted by using the reformulation of [15, Section III]. The latter is given below:

$$
\begin{aligned}
& \boldsymbol{P 4}: \mathbf{z}_{j}^{\star}=\underset{\mathbf{z}_{j}}{\operatorname{argmax}} Q_{j}\left(\mathbf{z}_{j}\right)=\left(1+z_{d 1}^{j}\right)\left(1+z_{d 2}^{j}\right) \\
& \text { s.t. } \mathbf{z}_{j} \in \mathcal{S}=\mathcal{S}_{n} \cap \mathcal{S}_{c} \\
& \mathcal{S}_{n}=\left\{\mathbf{z}_{j} \mid \mathbf{z}_{j} \preceq \Gamma_{j}, \mathbf{p}_{j} \preceq \mathbf{p}_{\max }\right\} \\
& \mathcal{S}_{c}=\left\{z_{b s}^{j} \geq \gamma_{\min }^{j}, z_{d 1}^{j} \geq 0, z_{d 2}^{j} \geq 0\right\}
\end{aligned}
$$

wherein $\mathbf{z}_{j}=\left(z_{d 1}^{j}, z_{d 2}^{j}, z_{b s}^{j}\right)$ and $\mathbf{z}_{j}^{\star}=\left(z_{d 1}^{j, \star}, z_{d 2}^{j, \star}, z_{b s}^{j, \star}\right)$. It is easily verified that the new objective function $Q_{j}\left(\mathbf{z}_{j}\right)$ is an increasing function with $\mathbf{z}_{j}$, and that $\mathcal{S}_{n}$ and $\mathcal{S}_{c}$ are respectively normal and co-normal sets. Hence $\boldsymbol{P} 4$ is $\mathrm{MO}$ problem and it can be solved in exponential time using the outer polyblock approximation algorithm (OPA) described in [15] and reported in [14, Algorithm 3]. The OPA algorithm solves the rate maximization problem by constructing a sequence of shrinking polyblocks that eventually reaches the global optimal point.

Since the objective function of $\boldsymbol{P 4}$ is increasing with $\mathbf{z}_{j}$, the optimal solution $Z_{j}^{\star}$ must occur at a place where $\mathbf{z}_{j}=\Gamma_{j}$. Accordingly, the optimal solution of (10) denoted by $\mathbf{p}_{j}^{\star}$ can be recovered by solving the following linear equations system:

$$
\left\{\begin{array}{l}
P_{d 1}^{j} \eta z_{d 1}^{j, \star}-P_{d 2}^{j} g_{d}^{j}+P_{c}^{j} h_{c, d 1}^{j} z_{d 1}^{j, \star}+z_{d 1}^{j, \star} N_{0}=0 \\
-P_{d 1}^{j} g_{d}^{j}+P_{d 2}^{j} \eta z_{d 2}^{j, \star}+P_{c}^{j} h_{c, d 2}^{j} z_{d 2}^{j, \star}+z_{d 2}^{j, \star} N_{0}=0 \\
P_{d 1}^{j} h_{d 1, b s}^{j} z_{d 3}^{j, \star}+P_{d 2}^{j} h_{d 2, b s}^{j} z_{d 3}^{j, \star}-P_{c}^{j} g_{c, b s}^{j}+z_{d 3}^{j, \star} N_{0}=0 .
\end{array}\right.
$$

As a result, solving $\boldsymbol{P 4}$ for all $j \in\{1,2, \ldots, M\}$ yields to the optimal solution of $\boldsymbol{P 3}$. Due to the exponential complexity of OPA, in the next section, we develop an analytical framework to compute the global optimal point with low complexity.

\section{GALEN'S FRAMEWORK}

Despite the interest of MO optimization theory, the latter converges to the optimal solution at the expense of exponential complexity. In this section, we provide a mathematical framework named GALEN which finds the optimal solution of $\boldsymbol{P} \mathbf{2}$ in an efficient way, by analyzing the geometric structure of the feasible set $\Phi$.

\footnotetext{
${ }^{2}$ In this paper we use $\mathbf{x} \preceq \mathbf{y}$ to denote that the vector $\mathbf{y} \in \mathbb{R}^{N}$ is greater than or equal to $\mathbf{x} \in \mathbb{R}^{N}$ in a component-wise manner.
} 


\section{A. Geometric representation of $\Phi$}

The feasible set $\Phi$ of each couple $\left(D 2 D_{j}, C U_{j}\right)$ can be rewritten as follows:

$$
\begin{aligned}
& \Phi=\left\{P_{c}^{j} g_{c, b s}^{j}-\gamma_{\min }^{j}\left(P_{d 1}^{j} h_{d 1, b s}^{j}+P_{d 2}^{j} h_{d 2, b s}^{j}+N_{0}\right) \geq 0\right. \\
& \left.0 \leq P_{i}^{j} \leq P_{\max }^{i}, i \in\{d 1, d 2, c\}\right\}
\end{aligned}
$$

Equations (14) and (15) respectively represent the QoS constraint of $C U_{j}$ and the power constraints of the couple $\left(D 2 D_{j}, C U_{j}\right)$. Denote by $\mathcal{P}_{c}$ the equality case of (14), i.e., the QoS plane. Moreover, let $\mathcal{P}_{i}^{\max }\left(\mathcal{P}_{i}^{0}\right)$ be the maximum (minimum) power planes, i.e., $P_{i}^{j}=P_{\max }^{i}\left(P_{i}^{j}=0\right)$ where $i \in\{c, d 1, d 2\}$. Hence, in the three dimensional space $\left(P_{d 1}^{j}, P_{d 2}^{j}, P_{c}^{j}\right), \Phi$ can be represented as the intersection of $\mathcal{P}_{c}$ with $\mathcal{P}_{i}^{0}$ and $\mathcal{P}_{i}^{\max }$, where $i=\{c, d 1, d 2\}$.

It can be observed that $\mathcal{P}_{i}^{\max }\left(\mathcal{P}_{i}^{0}\right)$ with $i \in\{d 1, d 2, c\}$, are fixed in the 3D-space and they form a cuboid shape as shown in Fig.2a-2e. However, the plane $\mathcal{P}_{c}$ varies according to the channel situation of $\left(D 2 D_{j}, C U_{j}\right)$ and the required rate of $C U_{j}$. Thus, $\Phi$ may have different possible shapes as shown in Fig.2a-2e.

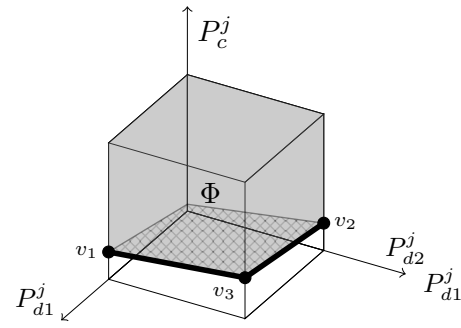

(a)

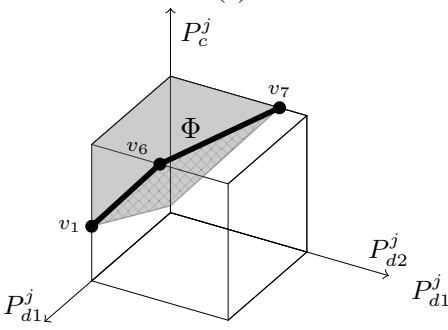

(c)

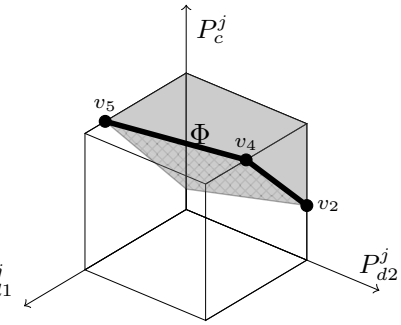

(b)

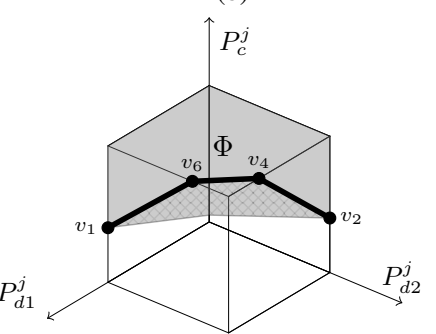

(d)

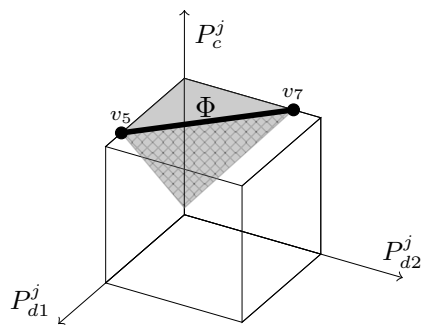

(e)

Fig. 2. The possible shapes of the feasible set $\Phi$. ( $\mathscr{P}_{c}$

Fig.2a-2c shows the cases where $\mathscr{P}_{c}$ has intersection with two maximum power planes and two minimum power planes. Fig.2b shows the case where $\mathcal{P}_{c}$ intersects all the maximum and the minimum power planes, while Fig.2e shows the case where $\mathscr{P}_{c}$ intersects the plane $\mathcal{P}_{c}^{\max }, \mathcal{P}_{d 1}^{0}$ and $\mathcal{P}_{d 2}^{0}$. The thick lines in the above figures represent the intersection lines of $\mathcal{P}_{c}$ with the maximum power planes. The corner of these lines are denoted as $v_{i}$ with $i=\{1,2, \ldots, 7\}$. Moreover, we define the follows $v_{1} \triangleq \mathcal{P}_{c} \cap \mathcal{P}_{d 1}^{\max } \cap \mathcal{P}_{d 2}^{0} ; v_{2} \triangleq \mathcal{P}_{c} \cap \mathcal{P}_{d 2}^{\max } \cap \mathcal{P}_{d 1}^{0} ; v_{3} \triangleq$ $\mathcal{P}_{c} \cap \mathcal{P}_{d 1}^{\max } \cap \mathcal{P}_{d 2}^{\max } ; v_{4} \triangleq \mathcal{P}_{c} \cap \mathcal{P}_{d 2}^{\max } \cap \mathcal{P}_{c}^{\max } ; v_{5} \triangleq \mathcal{P}_{c} \cap \mathcal{P}_{c}^{\max } \cap \mathcal{P}_{d 2}^{0} ;$ $v_{6} \triangleq \mathcal{P}_{c} \cap \mathcal{P}_{d 1}^{\max } \cap \mathcal{P}_{c}^{\max }$; and $v_{7} \triangleq \mathcal{P}_{c} \cap \mathcal{P}_{c}^{\max } \cap \mathcal{P}_{d 1}^{0}$. Thus the coordinate of these vertices can be expressed as follows:

$$
\begin{aligned}
& v_{1}=\left(P_{\max }^{d 1} ; 0 ;\left(P_{\max }^{d 1} h_{d 1, b s}^{j}+N_{0}\right) \gamma_{\min }^{j} / g_{c, b s}^{j}\right) \\
& v_{2}=\left(0 ; P_{\max }^{d 2} ;\left(P_{\max }^{d 2} h_{d 2, b s}^{j}+N_{0}\right) \gamma_{\min }^{j} / g_{c, b s}^{j}\right) \\
& v_{3}=\left(P_{\max }^{d 1} ; P_{\max }^{d 2} ;\left(P_{\max }^{d 1} h_{d 1, b s}^{j}+P_{\max }^{d 2} h_{d 2, b s}^{j}+N_{0}\right) \gamma_{\min }^{j} / g_{c, b s}^{j}\right) \\
& v_{4}=\left(\left(P_{\max }^{c} g_{c, b s}^{j} / \gamma_{\min }^{j}-P_{\max }^{d 2} h_{d 2, b s}^{j}-N 0\right) / h_{d 1, b s}^{j} ; P_{\max }^{d 2} ; P_{\max }^{c}\right) \\
& v_{5}=\left(\left(P_{\max }^{c} g_{c, b s}^{j} / \gamma_{\min }^{j}-N 0\right) / h_{d 1, b s}^{j} ; 0 ; P_{\max }^{c}\right) \\
& v_{6}=\left(P_{\max }^{d 1} ;\left(P_{\max }^{c} g_{c, b s}^{j} / \gamma_{\min }^{j}-P_{\max }^{d 1} h_{d 1, b s}^{j}-N 0\right) / h_{d 2, b s}^{j} ; P_{\max }^{c}\right) \\
& v_{7}=\left(0 ;\left(P_{\max }^{c} g_{c, b s}^{j} / \gamma_{\min }^{j}-N 0\right) / h_{d 2, b s}^{j} ; P_{\max }^{c}\right)
\end{aligned}
$$

\section{B. Optimal power allocation}

Denote by $\Omega=\left\{\overline{v_{1} v_{3}} ; \overline{v_{2} v_{3}} ; \overline{v_{4} v_{5}} ; \overline{v_{6} v_{7}} ; \overline{v_{4} v_{6}} ; \overline{v_{5} v_{7}}\right\}$ the set of the intersection lines between $\mathcal{P}_{c}$ and the maximum power planes. The following lemma shows that the optima can be searched only in $\Omega$ instead of $\Phi$. Here it is worthy mentioning that $\Omega$ is an exclusive set, i.e., not all its members occurs at the same time as shown in Fig.2a-2e.

Lemma 1. The optimal power vector $\boldsymbol{p}_{j}^{\star}$ has at least one power bounded by the maximum power constraint and it lies on the $Q o S$ plane $\mathcal{P}_{c}$.

Proof: For any scaling parameter $\mu>1$ and a power vector $\mathbf{p}_{j}=\left(P_{d 1}^{j}, P_{d 2}^{j}, P_{c}^{j}\right) \in \Phi$ we have:

$$
\begin{aligned}
Q_{j}\left(\mu \mathbf{p}_{j}\right)= & {\left[\left(1+\frac{P_{d 2} g_{d}}{P_{c} g_{c, d 1}+\eta P_{d 1}+\sigma_{N}^{2} / \mu}\right) \times\right.} \\
& \left.\left(1+\frac{P_{d 1} g_{d}}{P_{c} g_{c, d 2}+\eta P_{d 2}+\sigma_{N}^{2} / \mu}\right)\right]>Q_{j}\left(\mathbf{p}_{j}\right)
\end{aligned}
$$

The power constraints defined in (15) imply that the maximum $\mu P_{i}^{j}$ is $P_{\max }^{i}, i \in\{d 1, d 2, c\}$ (due to inequality in (16)). Hence, the optimal point $\mathbf{p}_{j}^{\star}$ can be achieved only if at least one user is transmitting with the maximum allowed power.

On the other hand, since $Q_{j}$ monotonically decreases with $P_{c}^{j}$, the maximum $R_{f d}^{j}$ occurs when $C U_{j}$ transmits with the minimum required power. Thus, $\mathbf{p}_{j}^{\star}$ must reside on the plane $\mathcal{P}_{c}$. As a result, the optimal points must reside in $\Omega$

Lemma 1 reduces the feasible set $\Phi$ to $\Omega$, and thus the optimal point can be obtained by solving the power allocation problem defined in (10) over the different points of $\Omega$.

Being on $\overline{v_{1} v_{3}}$ ( see Fig.2a) or $\overline{v_{1} v_{6}}$ ( see Fig.2b and Fig.2c) implies that $P_{d 1}^{j}=P_{\max }^{d 1}$ and $P_{c}^{j}=\left(P_{\max }^{d 1} h_{d 1, b s}^{j}+P_{d 2}^{j} h_{d 2, b s}^{j}+\right.$ $\left.N_{0}\right) \frac{\gamma_{\min }^{j}}{g_{c, b s}^{j}}$. In such case, the objective function of (10) reduces to

$$
Q_{j}\left(\mathbf{p}_{j}\right)=\left(1+\frac{P_{d 2}^{j}}{P_{d 2}^{j} a_{0}+a_{1}}\right)\left(1+\frac{P_{\max }^{d 1}}{P_{d 2}^{j} a_{2}+a_{3}}\right)
$$


where the constant coefficients in the above equation are defined as

$$
\begin{aligned}
& a_{0}=\frac{\gamma_{\min }^{j} h_{d 2, b s}^{j} h_{c, d 1}^{j}}{g_{c, b s}^{j} g_{d}^{j}} ; a_{2}=\frac{\eta g_{c, b s}^{j}+\gamma_{\min }^{j} h_{d 2, b s}^{j} h_{c, d 2}^{j}}{g_{c, b s}^{j} g_{d}^{j}} \\
& a_{1}=\frac{P_{\max }^{d 1}}{g_{d}^{j}}\left(\eta+\frac{\gamma_{\min }^{j} h_{d 1, b s}^{j} h_{c, d 1}^{j}}{g_{c, b s}^{j}}\right)+\frac{N_{0}}{g_{d}^{j}}\left(1+\frac{\gamma_{\min }^{j} h_{c, d 1}^{j}}{g_{c, b s}^{j}}\right) \\
& a_{3}=\frac{\gamma_{\min }^{j} P_{\max }^{d 1} h_{d 1, b s}^{j} h_{c, d 2}^{j}}{g_{c, b s}^{j} g_{d}^{j}}+\frac{N_{0}}{g_{d}^{j}}\left(1+\frac{\gamma_{\min }^{j} h_{c, d 2}^{j}}{g_{c, b s}^{j}}\right) .
\end{aligned}
$$

Taking the first derivative of (17) w.r.t $P_{d 2}^{j}$ leads to the following:

$$
\frac{\partial(17)}{\partial P_{d 2}^{j}}=\frac{A_{1} P_{d 2}^{{ }^{2}}+2 B_{1} P_{d 2}^{j}+C_{1}}{D_{1}}
$$

where the constants $A, B, C$ and $D$ are given as follows:

$$
\begin{aligned}
& A_{1}=\left(a_{2}{ }^{2} a_{1}-P_{\max }^{d 1} a_{0} a_{2}\left(a_{0}+1\right)\right) ; \\
& B_{1}=-\left(P_{\max }^{d 1} a_{0}-a_{3}\right) a_{2} a_{1} ; C_{1}=P_{\max }^{d 1}\left(a_{1} a_{3}-a_{1}^{2} a_{2}\right)+a_{1} a_{3}^{2} ; \\
& D_{1}=\left(P_{d 2}^{j} a_{0}+a_{1}\right)^{2}\left(P_{d 2}^{j} a_{2}+a_{3}\right)^{2} .
\end{aligned}
$$

Since $D_{1}$ is always positive, a possible optima of (17) would be the solution of $A_{1} P_{d 2}^{j}{ }^{2}+2 B_{1} P_{d 2}^{j}+C_{1}$. Denote such optima point as $e_{1}$, the coordinates of $e_{1}$ are given by:

$$
e_{1}=\left(\begin{array}{c}
P_{\max }^{d 1} ; \frac{1}{A_{1}}\left(-B_{2} \pm \sqrt{B_{1}{ }^{2}-A_{1} C_{1}}\right) \\
\gamma_{\min }^{j}\left(P_{\max }^{d 1} h_{d 1, b s}^{j}+P_{d 2, e_{1}}^{j} h_{d 2, b s}^{j}+N_{0}\right) / g_{c, b s}^{j}
\end{array}\right)
$$

with $P_{d 2, e_{1}}^{j}$ being the $P_{d 2}^{j}$ abscissa of $e_{1}$.

The above solution does not always provide a global maxima of (17), since their is no guarantee that the second derivative is always negative. Moreover, $e_{1}$ may not be always a feasible point( since it may lies outside $\overline{v_{1} v_{3}}$ or $\overline{v_{1} v_{6}}$, or it might be an imaginary point). Accordingly, the global maxima of (17) would be either one of the corner points of the line $\overline{v_{1} v_{3}}\left(\overline{v_{1} v_{6}}\right)$, i.e., $v_{1}$ or $v_{3}\left(v_{6}\right)$, or the extreme point $e_{1}$ (if $e_{1}$ is feasible).

In the same manner, the optimal point of (10) on the line $\overline{v_{2} v_{3}}$ as in Fig.2a ( or $\overline{v_{2} v_{4}}$ as in Fig.2b and Fig.2d) is either one of the corner points $v_{2}$ or $v_{3}\left(v_{4}\right)$ or an extreme point $e_{2}$ which lies on $\overline{v_{2} v_{3}}\left(\overline{v_{2} v_{4}}\right)$ and has the following coordinate:

$e_{2}=\left(\begin{array}{c}\frac{1}{A_{2}}\left(-B_{2} \pm \sqrt{B_{2}^{2}-A_{2} C_{2}}\right) ; \\ P_{\max }^{d 2} ; \gamma_{\min }^{j}\left(P_{d 1, e_{2}}^{j} h_{d 1, b s}^{j}+P_{\max }^{d 2} h_{d 2, b s}^{j}+N_{0}\right) / g_{c, b s}^{j}\end{array}\right)$,

where the constants $A_{2}, B_{2}$, and $C_{2}$ are given by

$$
\begin{aligned}
& A_{2}=\left(b_{2}^{2} b_{1}-P_{\max }^{d 2} b_{0} b_{2}\left(b_{0}+1\right)\right) ; \\
& B_{2}=\left(b_{3}-P_{\max }^{d 2} b_{0}\right) b_{2} b_{1} ; C_{2}=P_{\max }^{d 1}\left(b_{1} b_{3}-b_{1}^{2} b_{2}\right)+b_{1} b_{3}^{2},
\end{aligned}
$$

and the constant coefficients $b_{0}, b_{1}, b_{2}$, and $b_{3}$ are given by

$$
\begin{aligned}
& b_{0}=\frac{\gamma_{\min }^{j} h_{d 1, b s}^{j} h_{c, d 2}^{j}}{g_{c, b s}^{j} g_{d}^{j}} ; b_{2}=\frac{\eta g_{c, b s}^{j}+\gamma_{\min }^{j} h_{d 1, b s}^{j} h_{c, d 1}^{j}}{g_{c, b s}^{j} g_{d}^{j}} \\
& b_{1}=\frac{P_{\max }^{d 2}}{g_{d}^{j}}\left(\eta+\frac{\gamma_{\min }^{j} h_{d 2, b s}^{j} h_{c, d 2}^{j}}{g_{c, b s}^{j}}\right)+\frac{N_{0}}{g_{d}^{j}}\left(1+\frac{\gamma_{\min }^{j} h_{c, d 2}^{j}}{g_{c, b s}^{j}}\right) \\
& b_{3}=\frac{\gamma_{\min }^{j} P_{\max }^{d 2} h_{d 2, b s}^{j} h_{c, d 1}^{j}}{g_{c, b s}^{j} g_{d}^{j}}+\frac{N_{0}}{g_{d}^{j}}\left(1+\frac{\gamma_{\min }^{j}}{g_{c, b s}^{j}} h_{c, d 1}^{j}\right) .
\end{aligned}
$$

Similarly, the optimal point of (10) on the line $\overline{v_{6} v_{4}}$ as in Fig.2d ( or $\overline{v_{5} v_{7}}$ as in Fig.2e) is either one of the corner points $v_{4}$ or $v_{6}\left(v_{5}\right.$ or $\left.v_{7}\right)$ or an extreme point $e_{3}$ which lies on $\overline{v_{6} v_{4}}$ $\left(\overline{v_{5} v_{7}}\right)$ and has the following coordinate

$$
e_{3}=\left(\begin{array}{l}
\frac{1}{A_{3}}\left(-B_{3} \pm \sqrt{B_{3}^{2}-A_{3} C_{3}}\right) ; \\
\frac{1}{h_{d 2, b s}^{j}}\left(\frac{P_{\max }^{c} g_{c, b s}^{j}}{\gamma_{\min }^{j}}-N_{0}-P_{d 1, e_{3}}^{j} h_{d 1, b s}^{j}\right) ; P_{\max }^{c}
\end{array}\right),
$$

where the constants $A_{3}, B_{3}$, and $C_{3}$ are given by

$$
\begin{aligned}
& A_{3}=\eta c_{3}\left(\eta-c_{2}\right)-c_{4}\left(c_{4}-1\right)\left(c_{0} c_{2}+c_{1} \eta\right) ; \\
& B_{3}=\left(\left(c_{2} c_{4}-c_{2}+\eta\right) c_{0}+\eta c_{1} c_{4}\right) c_{3} ; \\
& C_{3}=c_{3}\left(c_{0}\left(c_{0}+c_{1}\right)-\left(c_{0} c_{2}+c_{1} \eta\right) c_{3}\right) ;
\end{aligned}
$$

and the constant coefficients $c_{0}, c_{1}, c_{2}$, and $c_{3}$ are given by

$$
\begin{aligned}
& c_{0}=\frac{1}{g_{d}^{j}}\left(P_{\max }^{c} g_{c, d 1}+N_{0}\right) ; c_{1}=\frac{P_{\max }^{c} g_{c, b s}^{j}}{\gamma_{\min }^{j} h_{d 2, b s}^{j}}-\frac{N_{0}}{h_{d 2, b s}^{j}} ; \\
& c_{2}=\frac{h_{d 1, b s}^{j}}{h_{d 2, b s}^{j}} ; c_{4}=\frac{\eta h_{d 1, b s}^{j}}{h_{d 2, b s}^{j} g_{d}^{j}} ; \\
& c_{3}=\frac{P_{\max }^{c}}{g_{d}^{j}}\left(g_{c, d 2}+\frac{\eta g_{c, b s}}{\gamma_{\min }^{j} h_{d 2, b s}^{j}}\right)+\frac{N_{0}}{g_{d}^{j}}\left(1-\frac{\eta}{h_{d 2, b s}^{j}}\right) .
\end{aligned}
$$

Accordingly, the optimal point would be the point which gives higher rate. The following Theorem summarizes all the previous discussion.

Theorem 1. The optimal power vector $\boldsymbol{p}_{j}^{\star}$ can be searched only in the set $\mathcal{P}_{\text {opt }}$ defined as

$$
\mathcal{P}_{\text {opt }}=\left\{v_{1}, v_{2}, v_{3}, v_{4}, v_{5}, v_{6}, v_{7}, e_{1}, e_{2}, e_{3}\right\}
$$

Here we should emphasize that Theorem 1 reduces the feasible set from a three dimensional shape to a set of points with known coordinates. Hence, with GALEN the global optimal point can be obtained in one iteration on contrary to the MO algorithm which requires a high number of iterations. Moreover, GALEN provides a closed form expression of the optimal solution.

\section{NUMERICAL RESULTS}

In this section we aim to find the ultimate performance of a FD-D2D enabled cellular network. To that end, we consider a single cell network with radius $R$ and $M$ CUEs and D2D pairs. The CUs are equally sharing the uplink bandwidth and they are uniformly distributed in the cell. Moreover, each $D 2 D_{j}$ pair is uniformly distributed within a randomly located cluster with radius $r$, and is sharing the same resource of $C U_{j}$. All the simulation parameters are summarized in Table I. 
TABLE I

SIMULATION PARAMETERS

\begin{tabular}{|l|l|}
\hline Cell radius $(\mathrm{R})$ & $500 \mathrm{~m}$ \\
\hline Cluster radius(r) & $10 \mathrm{~m}, 20 \mathrm{~m}, 40 \mathrm{~m}, 80 \mathrm{~m}$ \\
\hline Number of CUs and D2D pairs $(\mathrm{M})$ & 15 \\
\hline Bandwidth of $C U_{j}\left(B_{j}\right)$ & $180 \mathrm{KHz}$ \\
\hline Noise power $\left(N_{0}\right)$ & $-114 \mathrm{dBm}$ \\
\hline Path-loss exponent $(\alpha)$ & 4 \\
\hline Maximum power of CUE and D2D users & $24 \mathrm{dBm}$ \\
\hline SINR requirement for CUs $\left(\gamma_{\text {min }}^{j}\right)$ & $\begin{array}{l}\text { uniform distributed in } \\
{[0,25] \mathrm{dB}}\end{array}$ \\
\hline SI cancellation factor $(\eta)$ & $-100,-90, \ldots,-50$ dB \\
\hline Multiple-path fading & $\begin{array}{l}\text { Exponential distribu- } \\
\text { tion with } l_{i j}^{-\alpha} \text { mean }\end{array}$ \\
\hline
\end{tabular}

\section{A. GALEN VS MO}

In this subsection we aim to validate GALEN by comparing its results with the well known monotonic optimization framework. Fig. 3 shows the variation of the D2D rate w.r.t the SI cancellation factor and the D2D cluster radius. Moreover, it compares the proposed power allocation GALEN with the MO method for different cluster radius and different SI cancellation factors. In this figure we consider 500 realizations each with $M=15$ CUEs and $M=15$ D2D pairs. At Each iteration we averaged the results of all the couples $\left(D 2 D_{j}, C U_{j}\right)$ and finally we averaged the results over all realizations. As it can be seen, the curves perfectly match in all cases. Moreover, Fig. 3 clearly shows that as the cluster radius $r$ increases, the average rate decreases. As expected, we can see that the average rate increases as the SI factor decreases.

Moreover, to highlight the importance of GALEN, we show in Fig. 4 the complexity of $\mathrm{MO}$ in terms of the required number of iterations to achieve the global optimality. As it can be seen, the MO theory requires $10^{4}$ to achieve the optimal point. However, from Theorem 2, we know that GALEN obtains the optimal solution by searching a set of ten points. This low complexity feature makes GALEN a perfect choice for power allocation. Moreover, it makes the analysis of a FD-D2D network easier. Fig. 4 also shows that the number of iterations declines with the increasing of the cluster radius. This is because when $r$ increases the average number of admitted D2D pairs decreases.

\section{B. $F D-D 2 D$ or $H D-D 2 D$}

Here we aim to investigate the performance of the FDD2D communication, and see if it always outperforms the HD-D2D mode. To that end, and for fair comparison with FD, we assume that in the HD mode the D2D users in each D2D pair are equally sharing the bandwith $B_{j}$. Therefore, the received SINR at the BS will remain the same as in (1). However, the received SINR at $D_{1}^{j}$ and $D_{2}^{j}$ will no longer contain the SI component (i.e. $\eta$ component). Accordingly, denote by $\Gamma_{h d, d 1}^{j}$ and $\Gamma_{h d, d 2}^{j}$ the received SINR at the D2D devices in the HD mode, we have $\Gamma_{h d, d 1}^{j}=\left.\Gamma_{d 1}^{j}\right|_{\eta=0}$ and $\Gamma_{h d, d 2}^{j}=\left.\Gamma_{d 2}^{j}\right|_{\eta=0}$. Consequently, the HD D2D rate $\left(R_{h d}^{j}\right)$ becomes $R_{h d}^{j}=\left.\frac{1}{2} R_{h d}^{j}\right|_{\eta=0}$. Based on that, by setting $\eta=0$, both GALEN and MO can be used to derive the global optimal

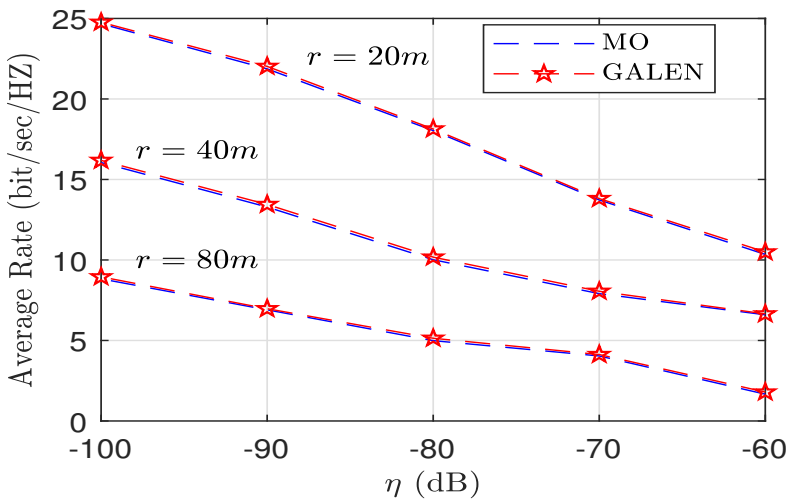

Fig. 3. Comparison of FD-D2D rate obtained from GALEN and from the MO theory.

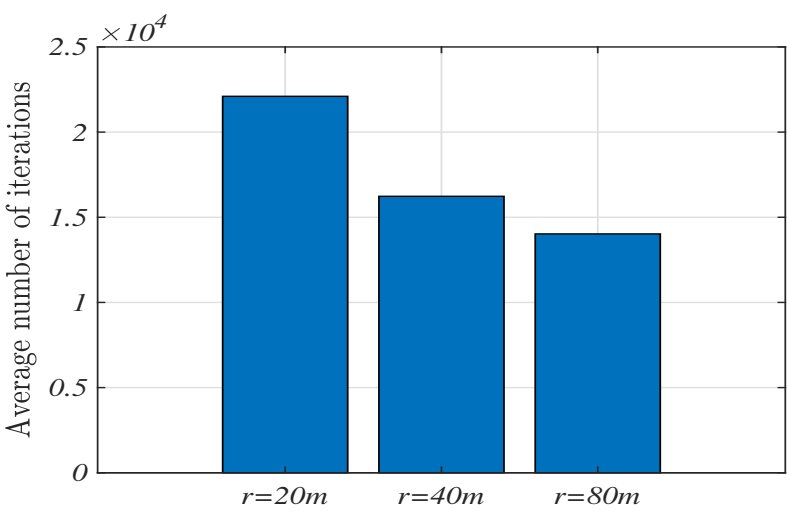

Fig. 4. The average number of iterations in the MO algorithm. solution of the rate maximization problem when the D2D users are operating in HD mode.

In Fig.5 we compare the FD-D2D performance with the HD-D2D in terms of the average D2D rate. In particular, Fig. 5 shows the variation of the D2D rate w.r.t the SI cancellation factor and for different D2D cluster radius. As it can be seen, at low self interference cancellation in the FD mode, the HD mode outperforms FD. In addition when the separation between the D2D users increases the FD mode needs higher SI cancellation to outperform the HD mode. As a result, the FD gain mainly appears when the D2D users are close to each other and they have high SI cancellation capability. Hence, to achieve the maximum gain from the D2D technology, a mode selection step must always be taken into consideration when allocating the resources for the users. The latter is out of scope of the paper and will be elaborated in the future.

\section{CONCLUSION}

This paper proposed two approaches to globally solve the power allocation problem of a FD-D2D network. The first one is based on the MO theory to the detriment of high complexity, while the latter, denoted GALEN, is a geometric-based approach with very low complexity. The simulation results have validated the simplified GALEN approach and have shown its low complexity feature. Moreover, simulation results have shown that the HD-D2D might outperform the FD-D2D in some situations. As a future work, the resource allocation 


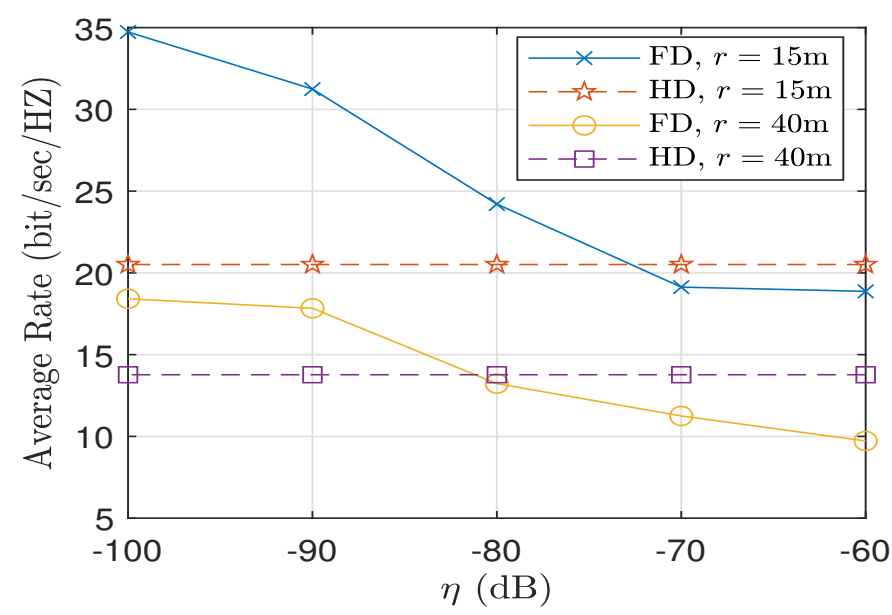

Fig. 5. Comparison of the FD-D2D rate and the HD-D2D rate.

which contains mode selection and channel assignment of a FD-D2D network will be considered. In addition, it might be interesting to analyze the maximization problem of the global cell rate, i.e., the rate of both $\mathrm{D} 2 \mathrm{D}$ and the cellular users.

\section{ACKNOWLEDGEMENT}

This work was partially funded through French National Research Agency (ANR) project WONG5 with grant agreement code: ANR-15-CE25-0005603. A part of this work was also carried out thanks to the STSM grant of COST Action CA15104 (IRACON) and CNRS (GdR-ISIS).

\section{REFERENCES}

[1] V. Cisco Mobile, "Cisco visual networking index: Global mobile data traffic forecast update, 2016-2021 white paper," 2017.

[2] K. Doppler, M. Rinne, C. Wijting, C. B. Ribeiro, and K. Hugl, "Deviceto-device communication as an underlay to lte-advanced networks," IEEE Communications Magazine, vol. 47, no. 12, 2009.

[3] A. Sabharwal, P. Schniter, D. Guo, D. W. Bliss, S. Rangarajan, and R. Wichman, "In-band full-duplex wireless: Challenges and opportunities," IEEE Journal on Selected Areas in Communications, vol. 32, no. 9, pp. 1637-1652, 2014.

[4] V. Tapio, "System scenarios and technical requirements for full-duplex concept," DUPLO, Project Deliverable, 2013.

[5] W. Cheng, X. Zhang, and H. Zhang, "Optimal power allocation for fullduplex d2d communications over wireless cellular networks," in 2014 IEEE Global Communications Conference, Dec 2014, pp. 4764-4769.

[6] B. Zuo, L. Jiang, C. He, and Z. Lian, "Power allocation optimization for full-duplex d2d communications underlaying cellular networks," in 2016 International Conference on Networking and Network Applications (NaNA). IEEE, 2016, pp. 103-108.

[7] K. T. Hemachandra, N. Rajatheva, and M. Latva-aho, "Sum-rate analysis for full-duplex underlay device-to-device networks," in 2014 IEEE Wireless Communications and Networking Conference (WCNC), April 2014, pp. 514-519.

[8] H. Chour, F.Bader, Y. Nasser, and O. Bazzi, "Full-duplex or half-duplex $\mathrm{d} 2 \mathrm{~d}$ mode? closed form expression of the optimal power allocation," in 25th International Conference on Telecommunication, 2018.

[9] S. Li, Q. Ni, Y. Sun, and G. Min, "Resource allocation for weighted sum-rate maximization in multi-user full-duplex device-to-device communications: Approaches for perfect and statistical csis," IEEE Access, vol. 5, pp. $27229-27241,2017$.

[10] P. D. Tao et al., "The dc (difference of convex functions) programming and dca revisited with dc models of real world nonconvex optimization problems," Annals of operations research, vol. 133, no. 1-4, pp. 23-46, 2005.

[11] Y. J. A. Zhang, L. Qian, J. Huang et al., "Monotonic optimization in communication and networking systems," Foundations and Trends $\AA$ in Networking, vol. 7, no. 1, pp. 1-75, 2013.
[12] H. Tuy, "Monotonic optimization: Problems and solution approaches," SIAM Journal on Optimization, vol. 11, no. 2, pp. 464-494, 2000.

[13] T. Riihonen, S. Werner, and R. Wichman, "Mitigation of loopback selfinterference in full-duplex mimo relays," IEEE Transactions on Signal Processing, vol. 59, no. 12, pp. 5983-5993, 2011.

[14] Y. J. A. Zhang, L. Qian, J. Huang et al., "Monotonic optimization in communication and networking systems," Foundations and Trends $®$ in Networking, vol. 7, no. 1, pp. 1-75, 2013.

[15] L. P. Qian, Y. J. Zhang, and J. Huang, "Mapel: Achieving global optimality for a non-convex wireless power control problem," IEEE Transactions on Wireless Communications, vol. 8, no. 3, pp. 1553-1563, 2009. 